\title{
A patient-reported questionnaire developed in a German early arthritis cohort to assess periodontitis in patients with rheumatoid arthritis
}

\author{
Johanna Callhoff ${ }^{*} \mathbb{D}$, Thomas Dietrich², Mariya Chubrieva' ${ }^{1}$, Jens Klotsche ${ }^{1}$ and Angela Zink ${ }^{1,3}$
}

\begin{abstract}
Background: The aim of this study was to develop a patient-reported questionnaire that is suitable to detect periodontitis (PD) in patients with rheumatoid arthritis (RA).

Methods: A self-reported questionnaire containing 12 items potentially relevant to PD and dentists' semiquantitative assessment of PD (no/mild/moderate/severe) was obtained from 353 patients from an early arthritis cohort. Available radiographs $(n=253)$ and blinded assessment of 3 independent dentists were used for validation. By defining the dentists' assessment as the reference standard, relevant questionnaire items were identified with factor analysis methods. Receiver operator characteristic (ROC) plots were used to determine sensitivities and specificities to detect PD in varying severity. Ordinal regression models were used to determine the coefficients for the final score.

Results: Seventy percent had at least mild PD. The items from the questionnaire correlating best with the dentists' assessment were selected for a final 6-item score (number of teeth, gum pockets, receding gums, loose teeth, receding jaw bone and tooth extractions and age). For the detection of any/moderate/severe PD, the bias-corrected areas under the curve (AUC) were $0.81 / 0.83 / 0.90$. Sensitivity to detect mild PD was $85 \%$ and specificity $57 \%$. Very high specificity was achieved for the detection of severe PD with $99 \%$ at the cost of low sensitivity (28\%).

Conclusions: This patient-reported six-item score has moderate diagnostic properties to study PD in RA patients in epidemiological settings. We propose to use the score as a measure of periodontitis without applying cut-off values.
\end{abstract}

Keywords: Periodontitis, Rheumatoid arthritis, Self-reported questionnaire, Validation

\section{Introduction}

Over the past years, the association between chronic periodontitis (PD) and rheumatoid arthritis (RA) has received considerable attention [1-5]. In a systematic review of studies on the association of PD and RA [6], Kaur et al. reported good evidence for the association between PD and tooth loss and attachment loss in patients with RA. They also discuss several models for the "interplay between PD and RA", which include the possibilities that periodontitis precedes RA, that there are

\footnotetext{
* Correspondence: johanna.callhoff@drfz.de

${ }^{1}$ Epidemiology Unit, German Rheumatism Research Centre, Charitéplatz 1, Berlin, Germany

Full list of author information is available at the end of the article
}

common underlying inflammatory pathways and that RA and PD exacerbate each other [6].

In a case-control study with 22 RA patients and 22 healthy controls, Wolff et al. confirmed evidence that patients with RA suffer from a higher risk of periodontal attachment loss [7]. Large epidemiological studies could help to gain further knowledge on the association of $\mathrm{PD}$ and parameters of disease activity in RA. However, it may not always be feasible to include the assessment of the periodontal status from trained study dentists in large epidemiological settings as was the case in the studies performed by Choi et al. [8] and Ayravainen et al. [3]. Therefore, a self-reported questionnaire would be helpful to assess PD in patients with RA. Several self-reported patient questionnaires have been developed in the past in

(C) The Author(s). 2019 Open Access This article is distributed under the terms of the Creative Commons Attribution 4.0 International License (http://creativecommons.org/licenses/by/4.0/), which permits unrestricted use, distribution, and 
various non-RA-specific populations with reasonable validity [9-12]. Coburn et al. [13] published a self-reported PD questionnaire that was evaluated in 617 patients with RA and osteoarthritis. This questionnaire included 6 questions on the periodontal status as well as sex, age, education and smoking behaviour and also showed moderate validity.

Taking into account the previous work by Dietrich et al. [11] and others $[9,10,12]$, our aim was to develop a simple patient-reported questionnaire for PD that can be used for studying the relationship of RA and PD in epidemiological settings and to validate it in a large cohort of patients with RA.

\section{Patients and methods}

\section{Early arthritis cohort}

Patients from the early arthritis cohort study Course And Prognosis of Early Arthritis (CAPEA) were asked to participate in this project. CAPEA is a prospective, multicentre, non-interventional, observational study in which patients were enrolled between 2010 and 2013 [14]. Eligible patients had arthritis for less than 6 months. They were consecutively enrolled in rheumatology clinics and practices in Germany and observed for 2 years in order to investigate the prognostic value of early symptoms for the development of a chronic course of disease. Ethical approval for CAPEA was obtained from the Ethics Committee of the Charite University Medicine, Berlin, in May 2009 with an amendment for the periodontitis project in May 2012.

\section{Patient-reported questionnaire on periodontitis}

All patients enrolled in CAPEA until January 2013 were sent a questionnaire including 12 questions about their PD status and other items considering dental replacement, comorbidities, current medication and pain. The questionnaire items were as follows: "number of teeth" (0-28), "receding jaw bone" ( 0 , no; 1 , yes), "receding gums" ( 0 , no; 1 , at up to 3 teeth; 2 , at 4 to 10 teeth; 3 , at over 10 teeth), "presence of gum pockets" ( 0 , none; 1 , at up to 3 teeth; 2 , at 4 to 10 teeth; 3 , at over 10 teeth), "loose teeth" ( 0 , no, never; 1 , I had loose teeth in the past; 2, yes, I currently have loose teeth), "tooth extractions because of inflammation and deep gum pockets" ( 0 , no; 1 , at up to 3 teeth; 2 , at 4 to 10 teeth; 3 , at more than 10 teeth), "more dentist visits because of inflammation than because of caries" ( 0 , no; 1 , yes), "more tooth/ gum problems than other persons of the same age and sex" (0, less than others or comparable to others; 1 , more than others; 2 , a lot more than others), "inflammation of the gums/bleeding" ( 0 , never; 1 , every few years; 2 , in many years; 3 , (nearly) every year), "magnitude of suffering from dental problems in total during the last 6 months" (0, not at all; 1 , a little bit; 2 , quite a bit; 3 , severe problems), "cold- or heat sensitivity" (0, no, never; 1 , yes, in the past; 2 , yes, currently) and "use of antibiotics to treat inflammation in the jaw bone" (0, never; 1 , once; 2 , two to five times; 3 , more than five times). Most of the questions were illustrated with pictures to demonstrate the appearance of a radiograph with receding jawbone for example. The questionnaire is available from the authors upon request.

\section{Dentists' assessment}

Patients were asked for the permission to contact their dentists. For all patients who returned a written consent, their dentists were then contacted by mail. They were asked to report whether or not the patient had been diagnosed with PD and to assess the PD status semiquantitatively with the possible answers "no", "mild (< $30 \%$ bone loss)", "moderate (30-50\% bone loss)" or "severe PD (> $50 \%$ bone loss)". Additionally, the number of teeth was reported. Furthermore, the dentists were asked to send any radiographs not older than 5 years for evaluation, if available.

The obtained radiographs were scored independently by three dentists at the School of Dentistry at the University of Birmingham, UK. The dentists were blinded to the clinical data of the patients. Disagreements were resolved by discussion. The confidence in the diagnosis of PD based on the available radiographs was rated as "certain", "pretty certain" or "uncertain".

The PD status reported by the patients' dentists was defined as the reference standard for PD for all analyses.

\section{Statistical analysis}

Correlations between the patient-reported items, the dentists' assessment and the blinded external assessment of the radiographs were analysed using Spearman's correlation coefficient. Confirmatory factor analysis was used to test the one-dimensional factor structure of the questionnaire. Items with similar content may result in correlated measurement errors [15] as indicated by large modification indices. Therefore, correlated residuals were assumed in the confirmatory factor model to avoid this method error. The evaluation of the model fit was based on the cut-offs as recommended by $\mathrm{Hu}$ and Bentler [16] (root mean square error of approximation (RMSEA) $\leq 0.06$, comparative fit index/Tucker-Lewis index (CFI/TLI) $\geq 0.9)$.

These items were used to calculate a final score for the detection of PD. Since age strongly correlates with the number of teeth and the probability to have PD, we always included age in the score [1].

The diagnostic properties of the score were evaluated by determining the sensitivity, specificity and the area under the receiver operator characteristic curve (AUC). Possible values for the AUC range from 0.5 to 1 : 0.5 
meaning a random classification of patients as having $\mathrm{PD}$ or not and 1 meaning perfect discrimination of the score between the groups. As the PD status was not assessed binary but with several levels of severity, different classifications of patients were performed. This resulted in three binary classifications of PD status: no versus mild/moderate/severe $\mathrm{PD}$, no/mild versus moderate/severe $\mathrm{PD}$ and no/mild/moderate versus severe $\mathrm{PD}$. To include all classifications of PD into a single model, an ordinal regression was performed so that it was possible to use the resulting score to assign patients to the most likely level of PD without having to choose which severity of PD should be detected.

\section{Correction for overoptimism}

The AUCs resulting from applying the model based on the whole dataset on the same data are likely too optimistic. We corrected for this overoptimism with bootstrap methodology. For 500 bootstrap samples of the size of the original dataset, models for the PD score were estimated. The resulting models were applied to both the original dataset and the respective bootstrap samples. Differences in the resulting AUCs were calculated, resulting in an estimator for the mean overoptimism. This estimator was subtracted from the original AUCs, resulting in bias-corrected AUCs. Additionally, the models based on the dentist's assessment of PD were applied to the subsample of patients with a radiographic assessment of PD, using this as the reference standard.

\section{Results}

\section{Study participation and baseline characteristics}

A total of 512 patients completed the patient questionnaire and gave permission to contact their dentists. We received 353 data sets with the dentist's assessments of the PD status and 253 data sets with additional radiographs. Radiographs of 4 patients were excluded due to insufficient quality. Figure 1 shows a flowchart of the respective patient numbers. The clinical characteristics at baseline of the different patient groups are shown in Table 1 . The subgroups were comparable to the CAPEA cohort except for a slightly higher mean number of teeth in the patients with available radiographs.

\section{PD assessments}

According to their dentists, $30 \%$ of the patients had no, $33 \%$ mild, 26\% moderate and 11\% severe PD. Of the 253 patients with radiographic evaluations, $23 \%$ had no, $25 \%$ mild, $29 \%$ moderate and $23 \%$ severe PD. For $41 \%$ of the patients, the three independent dentists rated the security of their PD assessment as certain; in $49 \%$ of cases, they were moderately certain; and in $11 \%$ of cases, they were uncertain, meaning that at least two dentists rated the PD status of the respective patients as uncertain.

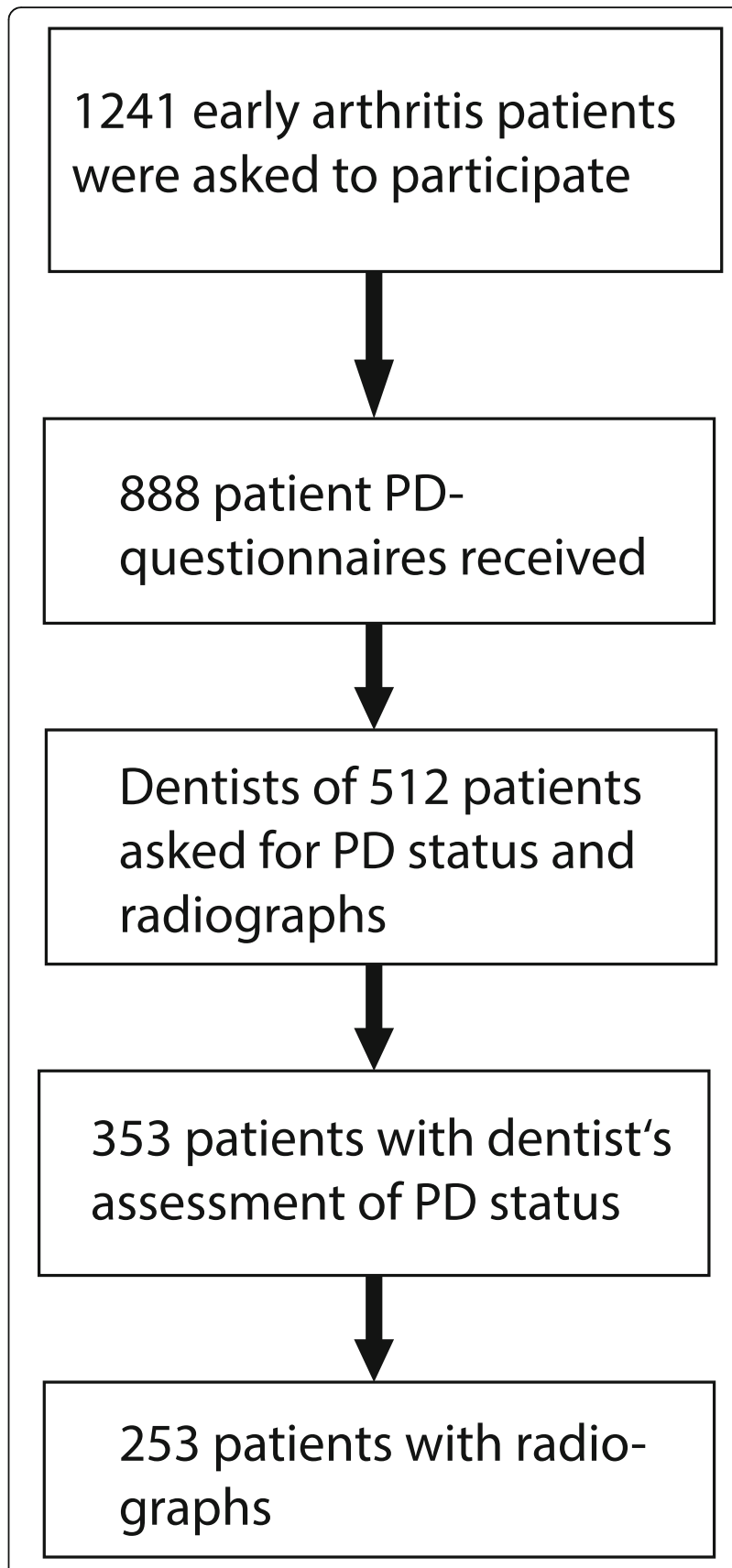

Fig. 1 Flowchart of participants who are included in the different analyses

Certainty was higher for the assessment of no or severe PD than for the assessment of mild or moderate PD.

The correlations between the patient-reported items and the dentists' assessments of PD are shown in Table 2. They were highest for the patient-reported number of teeth, receding jaw bone, receding gums, presence of gum pockets and loose teeth. Those items also correlated highest with the independent assessment of PD via radiographs. The strength of the correlation is only moderate with the highest correlation coefficient of - 
Table 1 Baseline characteristics

\begin{tabular}{lllll}
\hline Variable & $\begin{array}{l}\text { All CAPEA patients } \\
(n=1241)\end{array}$ & $\begin{array}{l}\text { PD module patients } \\
(n=512)\end{array}$ & $\begin{array}{l}\text { Patients with dentist's assessment } \\
(n=353)\end{array}$ & $\begin{array}{l}\text { Patients with radiograph } \\
(n=253)\end{array}$ \\
\hline Age, years & $56.2(14.3)$ & $56.3(14)$ & $55.8(13.3)$ & $55.8(13.3)$ \\
Sex, female & $65 \%(821)$ & $67 \%(575)$ & $64 \%(250)$ & $67 \%(170)$ \\
DAS28 ESR & $4.7(1.4)$ & $4.8(1.4)$ & $4.7(1.4)$ & $4.7(1.4)$ \\
ESR, mm/h & $31.2(23.4)$ & $31.8(23.7)$ & $31(22.9)$ & $30(22)$ \\
CRP, mg/l & $18.9(31.9)$ & $17.8(28.4)$ & $16.7(22.3)$ & $15.5(21.4)$ \\
Number of teeth & $19.2(9.6)$ & $19.4(9.6)$ & $20.8(8.3)$ & $21.1(7.7)$ \\
SJC28 & $6(5.2)$ & $6(5.2)$ & $5.6(4.8)$ & $5.4(4.7)$ \\
TJC28 & $9.7(6.2)$ & $9.5(6)$ & $9.2(5.9)$ & $9.1(5.8)$ \\
RF positive & $43 \%(539)$ & $43 \%(370)$ & $44 \%(169)$ & $40 \%(103)$ \\
Anti-CCP positive & $39 \%(493)$ & $38 \%(331)$ & $40 \%(157)$ & $36 \%(93)$ \\
Currently smoking & $33 \%(413)$ & $29 \%(254)$ & $27 \%(105)$ & $25 \%(65)$
\end{tabular}

Baseline characteristics of all patients from the early arthritis cohort CAPEA, patients who completed the PD-module, patients with additional dentist's assessment of $\mathrm{PD}$ and patients with radiographs

CAPEA Course And Prognosis of Early Arthritis, PD periodontitis, RF rheumatoid factor, SJC swollen joint count, TJC tender joint count, ESR erythrocyte

sedimentation rate, DAS28 Disease Activity Score including 28 joints, CRP C-reactive protein

0.49 between the number of teeth and the dentist's assessment of PD.

\section{Selection of variables for the patient-reported PD score}

The factor structure of two models was tested by confirmatory factor analysis (CFA): (a) a one-factor model in which all items were included and (b) a one-factor model in which six items were included number of teeth, receding jaw bone, receding gums, presence of gum pockets, loose teeth, tooth extractions because of inflammation and deep gum pockets), which correlated among each other in preliminary analyses. The CFA including all questionnaire items did not result in an acceptable model fit $($ RMSEA $=0.096, \mathrm{CFI}=0.86, \mathrm{TLI}=$ 0.88 , WRMR $=0.96$ ). The model that included six selected items did not fit the data well $(\mathrm{RMSEA}=0.148$, $\mathrm{CFI}=0.89$, TLI $=0.82$, WRMR $=1.05)$. The modification indices suggested correlated residuals between the items "number of teeth" and "loose teeth" (modification indices $=26.5$ ). The resulting model with correlated residuals yielded an acceptable model fit (RMSEA $=0.06, \mathrm{CFI}=$ $0.94, \mathrm{TLI}=0.90, \mathrm{WRMR}=0.77$ ).

\section{Results from the binary models}

Six variables (number of teeth, receding jaw bone, receding gums, presence of gum pockets, loose teeth, tooth

Table 2 Correlations of questionnaire items with PD assessments

\begin{tabular}{llll}
\hline Patient questionnaire item & $\begin{array}{l}\text { Number of } \\
\text { missing values }\end{array}$ & $\begin{array}{l}\text { Spearman's corr. } \\
\text { with dentist's } \\
\text { assessment }\end{array}$ & $\begin{array}{l}\text { Spearman's corr. } \\
\text { with assessment } \\
\text { of radiographs }\end{array}$ \\
\hline Number of teeth & 22 & -0.49 & -0.40 \\
Receding jaw bone & 25 & 0.41 & 0.43 \\
Receding gums & 14 & 0.37 & 0.33 \\
Presence of gum pockets & 16 & 0.36 & 0.36 \\
Loose teeth & 9 & 0.27 & 0.36 \\
Tooth extractions because of inflammation and deep gum pockets & 13 & 0.26 & 0.22 \\
More dentist visits because of inflammation than because of caries & 12 & 0.19 & 0.17 \\
More tooth/gum problems than other persons of the same age and sex & 22 & 0.12 & 0.10 \\
Inflammation of the gums/bleeding & 8 & 0.11 & 0.08 \\
Magnitude of suffering from dental problems & 6 & 6 & 0.09 \\
Cold- or heat sensitivity & 6 & 0.08 & 0.08 \\
Use of antibiotics & 6.13
\end{tabular}

Correlations of questionnaire items with dentists' assessment of PD and with an independent assessment of PD via radiographs. Items in italics were found to be the most suitable to detect PD via factor analysis and are included in the final score 
extractions because of inflammation and deep gum pockets) were identified as having a prognostic value for PD. These variables and three additional demographic variables (age, sex and formal education) were used to calculate several binary scores for the assessment of PD. As there are three possible levels of disease severity, several results have to be considered for every possibility to classify patients with the score.

The item which correlated best with the different assessments of PD was the number of the remaining teeth. Therefore, the first proposed possibility to classify patients was to use only age and the self-reported number of the remaining teeth. This resulted in a sensitivity of $86 / 80 / 86 \%$ (no versus mild, moderate or severe $\mathrm{PD} /$ no or mild versus moderate or severe $\mathrm{PD} /$ no, mild or moderate versus severe PD), a specificity of $49 / 64 / 78 \%$ and an AUC of 0.73/0.78/0.86 (Table 3). For all models, the bias-corrected AUCs are only marginally lower. The AUCs of the models based on the dentist's assessment applied to the radiograph scoring data differ most from the original model for the two models in which severe PD is detected ( 0.66 versus 0.85 and 0.77 versus 0.90 ).

When using all items from the patient questionnaire that were identified as being useful for the classification of PD, all models improved the diagnostic properties compared to the simple model only using age and the number of teeth. The AUCs of these models range between 0.82 and 0.92 depending on the severity level of PD that shall be detected. The models including sex and formal education of the patients did not show more favourable properties than those only including age as a demographic variable (data not shown). Therefore, sex and formal education were not included in the score.

\section{Results from the ordinal regression model}

For the ordinal regression model, there was again a simple version with only the number of teeth and age, and one model including the five additional patient-reported items mentioned above. A likelihood ratio test showed that the model with the additional items is better than the simple version.

The following were the results for the score:

PD score $=2.8+0.033 \times$ age $+0.37 \times$ gum pockets + $0.30 \times$ receding gums $+0.45 \times$ loose teeth $+0.84 \times$ receding jaw bone $-0.40 \times$ tooth extractions $-0.12 \times$ number of teeth.

The cut-offs were 1.83 for mild PD, 3.91 for moderate PD and 6.26 for severe PD. For example, a patient with an age of 40 years, no reported tooth or gum problems and all 28 teeth would have a score of $2.8+1.32-3.36=$ 0.76 and would be classified as having no PD.

The following were the results for the simple version of the score:

PD score $($ simple version $)=2.5+0.036 \times$ age $-0.11 \times$ number of teeth. The corresponding cut-off values were 1.16 for mild PD, 2.88 for moderate PD and 4.91 for severe $P D$.

Table 4 shows the classification of the patients by the score compared to the reference standard. Patients with severe PD are only detected in less than $30 \%$ of the cases and most often classified as "moderate" (Table 5).

The longer version of the score had considerably more specificity for the detection of PD than the short version (57\% versus $40 \%$ ). It also had a higher sensitivity for detecting moderate or severe PD.

\section{Discussion}

A patient-reported questionnaire to detect PD in patients with RA was developed. Six patient-reported items were selected to build the age-adjusted score. The score had a fair sensitivity to detect mild, moderate or severe versus no PD and was very specific at excluding severe PD. Additionally, a simple score including only age and the number of teeth was evaluated. This score might be

Table 3 Diagnostic properties of logistic regression models

\begin{tabular}{|c|c|c|c|c|c|c|}
\hline \multirow[t]{2}{*}{ Model } & \multirow[t]{2}{*}{ Severity of detected PD } & \multicolumn{3}{|c|}{ Reference standard: dentist's assessment } & \multirow{2}{*}{$\begin{array}{l}\text { Bias- } \\
\text { corrected } \\
\text { AUC }\end{array}$} & \multirow[b]{2}{*}{$\begin{array}{l}\text { AUC of the } \\
\text { original } \\
\text { model } \\
\text { applied on } \\
\text { radiograph } \\
\text { scoring } \\
\text { data }\end{array}$} \\
\hline & & Sensitivity (\%) & Specificity (\%) & $A \cup C$ & & \\
\hline \multirow[t]{3}{*}{ Age + number of teeth } & Mild, moderate or severe versus no & 86.0 & 48.6 & 0.73 & 0.73 & 0.82 \\
\hline & Moderate or severe versus no or mild & 80.3 & 64.1 & 0.78 & 0.77 & 0.72 \\
\hline & Severe versus no, mild or moderate & 86.1 & 78.1 & 0.86 & 0.85 & 0.66 \\
\hline \multirow[t]{3}{*}{ Age +6 patient-reported items } & Mild, moderate or severe versus no & 64.2 & 88.5 & 0.82 & 0.81 & 0.88 \\
\hline & Moderate or severe versus no or mild & 72.8 & 80.7 & 0.85 & 0.83 & 0.83 \\
\hline & Severe versus no, mild or moderate & 96.6 & 81.5 & 0.92 & 0.90 & 0.77 \\
\hline
\end{tabular}

Sensitivities, specificities and AUCs to detect different levels of severity of PD in the simple model and in the model including six questionnaire items. The table also shows the AUCs of these models after correction for overoptimism with bootstrap methods and the AUCs of the models if the independent assessment of PD with radiographs is used as a reference standard 
Table 4 Concordance of score and dentist's assessment

\begin{tabular}{|c|c|c|c|c|c|}
\hline \multirow{2}{*}{$\begin{array}{l}\text { Classification } \\
\text { of PD by } \\
\text { score (age + } \\
6 \text { patient- } \\
\text { reported } \\
\text { items) }\end{array}$} & \multicolumn{5}{|c|}{ Dentist's assessment of PD } \\
\hline & No & Mild & Moderate & Severe & Total \\
\hline No & 55 & 29 & 2 & 0 & 86 \\
\hline Mild & 32 & 51 & 34 & 2 & 119 \\
\hline Moderate & 9 & 21 & 37 & 19 & 86 \\
\hline Severe & 0 & 0 & 1 & 8 & 9 \\
\hline Total & 96 & 101 & 74 & 29 & 300 \\
\hline
\end{tabular}

Comparison of classification of PD with the help of the PD score and the reference standard

useful if PD shall be studied in a setting where it is only feasible to ask one additional question considering PD. The simple version also had a high sensitivity for detecting at least mild PD and a very good specificity to exclude severe PD. The overall properties of the score with six patient-reported items were more favourable than those of the simple score. The AUCs of 0.81, 0.83 and 0.90 respectively for the detection of at least mild, at least moderate or severe PD were comparable to those found by Dietrich et al. [11], Gilbert and Litaker [10] and Taylor and Borgnakke [17] and a little bit higher than those found by Genco et al. [9] (AUC of 0.76 for the detection of severe PD in the myocardial infection periodontitis study). In contrast to these questionnaires for self-reported PD, our score does not include sex or formal education. This might be due to the different study collectives with this study only including RA patients and the other studies including patients from the general population those who had a myocardial infarction.

Compared to the questionnaire used by Coburn et al. [13] that was also evaluated on RA patients, our questionnaire had a better AUC for the detection of severe PD (0.79 versus 0.90). For the detection of mild or moderate PD, the AUCs were comparable. In the investigation by Coburn et al., patients received a full-mouth periodontal examination to determine their PD status, while in the CAPEA periodontitis project, the patients' dentists were asked to grade the severity of their patient's PD semiquantitatively. This shows that in a setting where the diagnosis for PD was more standardised and clinically evaluated, the resulting PD score still does not have more favourable properties.

The items included in this score had some overlap with those identified by Dietrich et al. [11] (loosening of teeth, dentist told patient had lost bone around his or her teeth) but also included the presence of gum pockets and bleeding gums which are not represented in the final models of Dietrich et al., Taylor and Borgnakke [17] and Gilbert and Litaker [10] (in Gilbert's score, a more general rating of "gum health" is included, though). There was also an overlap with the items used by Coburn et al. [13]. Items concerning bleeding gums, bone loss, deep pockets, loose teeth and oral surgery were also included in our questionnaire in a similar way. While "bleeding gums" was not included in the final PD score in our analysis; the parameter correlating best with PD in our analysis (number of teeth) was not included in Coburn et al.'s questionnaire.

One limitation of this study is that our reference standard to determine a patient's PD status is the report of the patient's individual dentist and was not evaluated by a study dentist. To validate the diagnosis, the radiographs were assessed externally by three independent dentists. If the PD score we developed is applied to these data, the AUCs are in the range of 0.77 to 0.88 which means that if an objective blinded assessment of PD is used as a reference standard, the questionnaire also performs reasonably well. While there were more male than female patients participating in the Coburn study, CAPEA patients form a representative sample of early arthritis patients in Germany with more female patients.

The sensitivity and specificity of the CAPEA PD questionnaire are reasonably good. In order to conduct large epidemiologic trials that further investigate the relationship between RA and PD, instruments with a high accuracy would be needed. The misclassification rate might be too high to assess the relationship between clinical features of RA and periodontal status, if the periodontal status is determined through a patient-reported questionnaire alone. This problem could partly

Table 5 Diagnostic properties for ordinal regression model

\begin{tabular}{|c|c|c|c|}
\hline Model & Severity of detected PD & Sensitivity (\%) & Specificity (\%) \\
\hline \multirow[t]{3}{*}{ Age + number of teeth } & Mild, moderate or severe versus no & 91.7 & 39.6 \\
\hline & Moderate or severe versus no or mild & 49.5 & 85.8 \\
\hline & Severe versus no, mild or moderate & 20.7 & 100 \\
\hline \multirow[t]{3}{*}{ Age +6 patient-reported items } & Mild, moderate or severe versus no & 84.8 & 57.3 \\
\hline & Moderate or severe versus no or mild & 63.1 & 84.8 \\
\hline & Severe versus no, mild or moderate & 27.6 & 99.6 \\
\hline
\end{tabular}

Sensitivities and specificities for the detection of different levels of PD with the score derived from the ordinal regression model 
be solved by using a continuous measure of PD instead of categorising patients to "no", "mild", "moderate" or "severe" PD. Using the PD score as a continuous measure would still allow investigating the correlation between the severity of clinical measures of RA and PD with less misclassification errors than when using the categorisation.

\section{Conclusions}

The CAPEA PD score can be used as a measure of PD in epidemiological settings. In a categorical analysis using cut-off values, researchers should keep in mind, however, that this score does show only moderate diagnostic properties. If high accuracy is not essential, the number of teeth and age alone can also be used as a simple measure for the detection of the frequency of PD in patients with RA.

\section{Abbreviations \\ Anti-CCP: Anti-citrullinated protein; AUC: Area under the receiver operator characteristic curve; CAPEA: Course And Prognosis of Early Arthritis; PD: Periodontitis; CFA: Confirmatory factor analysis; CFI/TLI: Comparative fit index/Tucker-Lewis index; CRP: C-reactive protein; DAS28: Disease Activity Score including 28 joints; ESR: Erythrocyte sedimentation rate; RF: Rheumatoid factor; RMSEA: Root mean square error of approximation SJC: Swollen joint count; TJC: Tender joint count; WRMR: Weighted root mean square residual}

\section{Acknowledgements}

The authors gratefully acknowledge the contribution of the participating patients and the consultant rheumatologists for recruiting and following the patients in the CAPEA cohort. We are deeply thankful for the support of the dentists participating in the study. This work was initiated by Gisela Westhoff, who designed the questionnaire and was the principal investigator of the study. She died in June 2014.

\section{Authors' contributions}

All authors drafted the article, revised it critically for important intellectual content and approved the final version to be published. JC had full access to all the data in the study and takes responsibility for the integrity of the data and the accuracy of the data analysis. AZ and TD were involved in the study conception and design. $\mathrm{MC}$ and $\mathrm{JC}$ were responsible for the acquisition of the data. JC, TD, MC, JK and AZ were involved in the analysis and interpretation of the data.

\section{Funding}

The CAPEA periodontitis project was funded by the Deutsche Rheuma-Liga Bundesverband e.V. CAPEA was funded by an unconditional grant from Pfizer.

\section{Availability of data and materials}

The datasets analysed during the current study are not publicly available, because we respect our patient's right to privacy. Consent for publication of the dataset has not been asked at the point of recruitment to the trial.

\section{Ethics approval and consent to participate}

Ethical approval was obtained from the Ethics Committee of the Charité University Medicine, Berlin

\section{Author details}

'Epidemiology Unit, German Rheumatism Research Centre, Charitéplatz 1, Berlin, Germany. ${ }^{2}$ Department of Oral Surgery, The School of Dentistry, University of Birmingham, Birmingham, UK. ${ }^{3}$ Department of Rheumatology and Clinical Immunology, Charité University Hospital, Berlin, Germany.

Received: 24 June 2019 Accepted: 16 August 2019

Published online: 29 August 2019

\section{References}

1. Detert J, Pischon N, Burmester GR, Buttgereit F. The association between rheumatoid arthritis and periodontal disease. Arthritis Res Ther 2010;12(5):218.

2. Tang Q, Fu H, Qin B, Hu Z, Liu Y, Liang Y, Zhou L, Yang Z, Zhong R. A possible link between rheumatoid arthritis and periodontitis: a systematic review and meta-analysis. Int J Periodontics Restorative Dent. 2017;37(1):79-86.

3. Ayravainen L, Leirisalo-Repo M, Kuuliala A, Ahola K, Koivuniemi R, Meurman $J \mathrm{H}$, Heikkinen AM. Periodontitis in early and chronic rheumatoid arthritis: a prospective follow-up study in Finnish population. BMJ Open. 2017;7(1): e011916.

4. da Silva AP, Bissada NF. Arthritis and periodontitis: an association debated for over two centuries. Curr Rheumatol Rev. 2016;12(3):202-7.

5. Fuggle NR, Smith TO, Kaul A, Sofat N. Hand to mouth: a systematic review and meta-analysis of the association between rheumatoid arthritis and periodontitis. Front Immunol. 2016:7:80

6. Kaur S, White S, Bartold PM. Periodontal disease and rheumatoid arthritis: a systematic review. J Dent Res. 2013;92(5):399-408.

7. Wolff B, Berger T, Frese C, Max R, Blank N, Lorenz HM, Wolff D. Oral status in patients with early rheumatoid arthritis: a prospective, case-control study. Rheumatology (Oxford). 2014;53(3):526-31.

8. Choi IA, Kim JH, Kim YM, Lee JY, Kim KH, Lee EY, Lee EB, Lee YM, Song YW. Periodontitis is associated with rheumatoid arthritis: a study with longstanding rheumatoid arthritis patients in Korea. Korean J Intern Med. 2016:31(5):977-86

9. Genco RJ, Falkner KL, Grossi S, Dunford R, Trevisan M. Validity of selfreported measures for surveillance of periodontal disease in two western New York population-based studies. J Periodontol. 2007;78(7 Suppl):1439-54.

10. Gilbert GH, Litaker MS. Validity of self-reported periodontal status in the Florida Dental Care Study. J Periodontol. 2007;78(7 Suppl):1429-38.

11. Dietrich T, Stosch U, Dietrich D, Schamberger D, Bernimoulin JP, Joshipura $K$. The accuracy of individual self-reported items to determine periodontal disease history. Eur J Oral Sci. 2005;113(2):135-40.

12. Blicher B, Joshipura K, Eke P. Validation of self-reported periodontal disease: a systematic review. J Dent Res. 2005;84(10):881-90.

13. Coburn BW, Sayles HR, Payne JB, Redman RS, Markt JC, Beatty MW, Griffiths GR, McGowan DJ, Mikuls TR. Performance of self-reported measures for periodontitis in rheumatoid arthritis and osteoarthritis. J Periodontol. 2015; 86(1):16-26.

14. Albrecht $K$, Callhoff J, Schneider M, Zink A. High variability in glucocorticoid starting doses in patients with rheumatoid arthritis: observational data from an early arthritis cohort. Rheumatol Int. 2015;35(8):1377-84.

15. Brown TA. Confirmatory factor analysis of the Penn State Worry Questionnaire: multiple factors or method effects? Behav Res Ther. 2003; 41(12):1411-26.

16. Hu LTB, M P. Fit indices in covariance structure modeling: sensitivity to underparameterized model misspecification. Psychol Methods. 1998;3: 424-53.

17. Taylor GW, Borgnakke WS. Self-reported periodontal disease: validation in an epidemiological survey. J Periodontol. 2007:78(7 Suppl):1407-20.

\section{Publisher's Note}

Springer Nature remains neutral with regard to jurisdictional claims in published maps and institutional affiliations.

Consent for publication

Not applicable. 